\title{
EPC 成本加酬金合同投资控制研究及思考
}

\section{Research and Thinking on EPC Cost Plus Reward Contract Investment Control \\ 田辉 ${ }^{1}$ 母晓红 $^{2}$ 高伟 $^{1}$}

Hui Tian ${ }^{1}$ Xiaohong $\mathrm{Mu}^{2}$ Wei Gao

1.中交第四航务工程勘察设计院有限公司

中国·广东 广州 510230；

2.中化泉州石化有限公司

中国・福建 泉州 362103

1. CCCC-FHDI Engineering Co.,Ltd.,

Guangzhou, Guangdong, 510230, China;

2. Zhonghua Quanzhou Petrochemical Co.,Ltd.,

Quanzhou, Fujian, 362103, China
【摘要】为了消化工程量变化和价格波动带来的风险, $\mathrm{EPC}$ 总承包商往往会将一定的风险 费包含在投标报价中, 这就势必导致中标价较高。如何在充分发挥 EPC 总承包设计与管理 优势的基础上, 实现建设投资总额的进一步减少, 真正做到提质增效, 是人们在进行项目 合同管理过程中值得深入研究的课题。

【Abstract】In order to digest the risks brought by engineering quantity changes and price fluctuations, EPC general contractors often include certain risk fees in the bid price, which will inevitably lead to higher bid price. How to give full play to EPC general contracting design and on the basis of management advantages, realizing the further reduction of total construction investment and truly improving quality and efficiency is a topic worthy of in-depth study in the process of project contract management.

【关键词】成本加酬金合同; 投资; 研究

【Keywords]cost plus remuneration contract; investment; research

【DOI】10.36012/etr.v2i1.1020

\section{1 引言}

考虑到目前工程项目的复杂性和专业的多样性, 为了更 好地管理项目，越来越多的大型项目业主在选择承包商时都 倾向于 EPC 总承包模式。一方面, EPC 总承包商的管理服务 能有效减轻业主方的管理压力,让专业的团队做专业的事情; 另一方面, 利用 EPC 总承包模式不仅能加快项目建设进程, 也能在建设初期有效地控制项目建设投资[1。

\section{2 成本加酬金合同模式}

为了研究引言中提出的课题,论文在 EPC 总承包发包时 尝试引入成本加酬金的合同价款模式，既确保项目按照 EPC 总承包的模式进行管理，又能建立承包人与发包人的造价联 动控制机制, 实现投资额的进一步减少。

目前，比较常用的成本加酬金合同模式大致可以分为两 大类,即成本补偿合同和目标激励合同[1]。成本补偿合同的合 同总价按照承包商实际成本加上固定数量或固定比例的酬金
来计算, 可以进一步划分为成本加固定酬金或成本加固定比 例酬金。

成本加固定酬金合同总价计算公式为:

$$
F=f\left(x_{i}\right)+C
$$

式中, $F$ 为承包商合同总价; $x_{i}$ 表示组成项目成本的各因子 (一般指设备材料费及建筑安装费) $C$ 为固定数额酬金。

成本加固定比例酬金合同总价计算公式为:

$$
F=f\left(x_{i}\right)+k f\left(x_{i}\right)
$$

式中, $F$ 为承包商合同总价; $x_{i}$ 表示组成项目成本的各因子 (一般指设备材料费及建筑安装费); $k$ 为固定比例, 承包商获 得的报酬为成本的一次函数。

成本补偿合同总价可以看成是成本因子的正比例函数, 成本越高, 其相应获得的报酬越高, 因此, 承包商并没有多少 意愿减少成本。相反, 为了弥补管理上的能力不足或者单纯为 了获得更好的收益, 承包商往往很保守, 将项目安全系数设置 的很高, 势必造成投资增加。为此,论文引入了另外一种合同 模式,即目标激励合同。 
目标激励合同的机制为底薪+提成, 即首先为项目设置一 个固定报酬的底薪, 然后设置一个成本目标值, 并将目标值作 为考核指标, 承包商围绕成本目标进行设计、采购与施工的策 划与组织, 并确保项目满足功能要求, 顺利通过验收并办理结 算 ${ }^{[3]}$ 。最终, 业主与承包商将结算的成本值与目标成本值进行 比较后,将结余作为双方分成的基础,承包商按照合同约定的 比例享受结余分成。

目标激励合同的合同额公式一般为:

$$
F=f\left(x_{i}\right)+C+k_{i}\left[f(a)-f\left(x_{i}\right)\right],(i=1,2,3, \cdots)
$$

式中, $F$ 为承包商的合同额; $x_{i}$ 表示组成项目成本的各因子 (一 般指设备材料费及建筑安装费 $) ; C$ 为固定酬金; $k_{i}(i=1,2,3, \cdots)$ 为分享系数; $f(a)$ 为业主为本项目设置的目标成本 (一般在投 标报价阶段公布, 即为投标控制价)。

在目标激励合同下,承包商一方面要加强自身管理,增强 履约能力, 争取完成项目既定的各项目标, 拿到固定酬金; 另 一方面, 承包商为了获得更好的经济收益, 必定会在目标成本 的引导下, 在设计的深化与优化、施工组织与协调、资源的投 人等方面加强造价控制, 在满足质量、安全及进度目标下, 充 分挖掘管理潜力, 为自己争取足够多的结余, 从而实现管理与 效率双丰收。

\section{3 目标激励合同案例研究}

某大型央企 $\mathrm{Z}$ 在福建地区投资建设炼油项目，在对该项 目加氢装置进行招标策划时, 为了早日发挥装置效益, 做到对 项目投资进行严格控制, 项目指挥部决定对加氢装置进行 EPC 总承包招标, 充分发挥 EPC 总承包商的管理才能和专业 优势, 并通过设置目标激励合同, 对该装置成本进行控制。

为了加快建设进程, 确保项目早日投产, 本装置项目在完 成方案设计后, 即启动 $\mathrm{EPC}$ 招标流程, 将设计、采购和施工的 全过程作为一个工作包发包, 并与中标单位 $\mathrm{Y}$ 签订 EPC 合 同。该合同费用构成如表 1 所示。

\section{表 1 合同费用结构表}

\begin{tabular}{c|c|c|c}
\hline 序号 & 费用名称 & 合同金额(万元) & 备注 \\
\hline 1 & 项目目标成本 & $f(a)$ & 实施过程中为 $f\left(x_{i}\right)$ \\
\hline 1.1 & 设备材料采购费 & $f\left(a_{1}\right)$ & 实施过程中为 $f\left(x_{1}\right)$ \\
\hline 1.2 & 建筑安装工程费 & $f\left(a_{2}\right)$ & 实施过程中为 $f\left(x_{2}\right)$ \\
\hline 2 & 固定比例酬金 & $C$ & \\
\hline 2.1 & 勘察设计费 & $C_{1}$ & \\
\hline 2.2 & 设备材料采购管理费 & $C_{2}$ & $C_{2}=k f\left(a_{1}\right)$ \\
\hline 2.3 & 建筑安装管理费 & $C_{3}$ & $C_{3}=k f\left(a_{2}\right)$ \\
\hline 3 & 结余分成 & $k f(a)-f\left(x_{i}\right)$ & $k$ 为常数 \\
\hline
\end{tabular}

合同约定, 在 $\mathrm{Z}$ 企业批复基础设计概算后, 项目目标成本 的调整: 当批复的基础设计概算中的 $f_{\text {批复 }}(a) \geqslant$ 签约合同价中
的 $f$ 合同 $(a)$, 合同价格表中的成本目标 $f$ 合同 $(a)$ 保持不变, 成本 的构成比例按照批复的基础设计进行相应调整; 当批复的基 础设计概算中的 $f_{\text {批复 }}(a)$ <签约合同价中的 $f_{\text {合同 }}(a)$, 合同价格表 中的成本目标 $f$ 合同 $(a)$ 调整为基础设计概算中批复的 $f_{\text {批复 }}(a)$, 并保持目标成本在批复的基础设计中的比例构成。

$\mathrm{EPC}$ 总承包商 $\mathrm{Y}$ 的合同总价=项目成本+固定酬金+结余 分成,即

$$
F=f\left(x_{i}\right)+C+\left\{\begin{array}{l}
k_{1}\left[f(a)-f\left(x_{i}\right)\right],\left(0 \leqslant f(a)-f\left(x_{i}\right) \leqslant 2000\right) \\
2000 k_{1}+k_{2}\left[f(a)-f\left(x_{i}\right)-2000\right], \\
\left(2000<f(a)-f\left(x_{i}\right) \leqslant 2000\right) \\
2000 k_{1}+1000 k_{2}+k_{3}\left[f(a)-f\left(x_{i}\right)-3000\right], \\
3000<f(a)-f\left(x_{i}\right)
\end{array}\right.
$$

式中, $F$ 为承包商获得的管理费; $x_{i}$ 表示组成项目成本的 各因素(本项目设备采购费与建筑安装工程费); $C$ 为固定酬金 (包含勘察设计费、设备材料费与建筑安装费固定比例酬金); $k_{i}(i=1,2,3, \cdots)$ 为分享系数; $f(a)$ 为业主为本项目设置的目标 成本。

本项目在执行过程中, $\mathrm{Z}$ 公司项目指挥部各部门通力协 作, 齐心协力, 在确保项目质量和安全的前提下, 项目最终高 标准通过验收, 满足项目使用要求。 $\mathrm{EPC}$ 总承包商 $\mathrm{Y}$ 通过合 理的设计深化与优化,加强设计与采购、施工之间的合理交 叉与融合, 同时, 在施工管理过程中, 强化施工的组织与协 调，合理划分工作界面，注意各工作面的流水施工。最终， EPC 承包商 $Y$ 获得了七千多万的结余分成,获得了良好的经 济效益。

Z 公司通过此模式, 不仅仅实现了既定的投资控制目标, 还节省了约五千万元的项目资金,达到了提质增效的目的。

\section{4 注意的问题及建议}

\section{1 注意的问题}

上述案例, 通过设置目标激励合同 (固定酬金+结余分成) 模式的 EPC 总承包, 合同双方都严格履行了合同义务,并最 终获得了较好的经济效益, 实现了项目管理的双赢。但不得 不说, 在项目策划及实施过程中, 还是有很多值得深入思考 的问题。

第一，如何在基础设计批复未完成前完成招标控制价的 合理设置是项目策划首先需要慎重考虑的问题。对于采用传 统工艺的装置项目，如何确保在控制价测算时保证工程量和 价款的合理; 对于采用新工艺的装置项目, 又如何完成控制价 的测算; 控制价是否能够较为真实的反映市场, 能不能得到市 场的响应（能不能得到投标单位的认可并吸引潜在投标人）, 
实验与研究 Experiments and Research

是否有流标的风险都是人们需要首先考虑和解决的问题, 是 项目能否按照此模式进行的关键步骤。

第二, 如何确保基础设计概算的合理性和准确性对于后 期项目合同执行至关重要。基础设计概算是整个项目造价的 上限值, 对于项目后期合同执行非常关键, 既是后期合同执行 的基础性数据, 能够为后期合同价款提供对比源, 又是合同执 行的控制性数据, 对项目结算起限制作用。费用构成是否涵盖 后期所有方面的工作, 经批准的各类型费用是否能准确覆盖 本项工作都直接影响项目后期执行, 其合理与否、准确与否直 接关系到项目后期合同执行是否顺利。

第三，基础设计批复与签约合同价中对应的目标成本不 一致的风险及合同价款调整机制该如何设定。在完成招标之 后，如果出现批复的基础设计概算对应的目标成本小于签约 合同价款中的目标成本，如何确保中标单位接受合同价款调 整, 中标单位能够接受合同价款的调整幅值及上限, 两个目标 成本相差太大会不会引起中标人放弃中标项目或者给合同履 行带来风险; 基础设计概算对应的目标成本大于签约合同成 本时, 按照什么原则调整签约合同价款和目标成本的构成比 例既能一定程度上控制投资, 又能较好地激发承办商的管控 潜力。

第四，重大设计变更的认定及成本目标的调整机制该如 何设定。如何就基础设计与方案设计的重大变更进行准确认 定, 并对其重大设计变更导致的采购及施工工艺的调整产生 的附加成本进行责任分担与费用分成, 如何将上述变化导致 的成本与成本目标调节机制结合起来，是项目实施过程中需 要重点考虑的问题。在本案例中, 业主最终结算与 EPC 总承 包就设计变更的认定及认定后涉及的控制目标的调整进行了 激烈交锋, 并经过了长时间的谈判, 最终认定追加项目目标成 本 400 万元, 并相应补偿 EPC 总承包商固定比例酬金, 并将 调整后的目标成本用以计算结余分成。

第五, 如何平衡成本与质量、安全之间的关系。项目在实 施过程中, 一方面, 是承包商为了追求经济效益, 一定程度上 减少对安全和质量管理的投入; 另一方面, 业主为了确保项目 后续的运行, 在质量管理过程中存在提高标准、增加工作范围 的现象 (业主质量及安全管理部门对合同费用构成并不熟悉), 势必会对项目合同执行造成困难。

第六,结算成本超过目标成本的处理机制该如何设定。项 目最终结算成本超过目标成本后如何对承包商的报酬进行约 定; 承包商结算成本超过目标成本的罚金标准该如何设置; 能 否建立成本超支后的费用分担机制; 如何确保成本超支后的 结算平顺性和合同的及时闭合。

\section{2 建议}

第一, 投标控制价四阶测算确认法。在基础设计尚未批复 以前 (一般完成方案设计), 为了最大程度逼近项目成本, 节约 项目投资, 首先系统收集及分析国际上类似项目的历史数据, 综合考虑装置参数、地质条件及物价上升的影响后, 折现成投 标阶段时点的控制价, 此为一阶控制价; 将一阶控制价作为原 始资料委托业内知名的咨询企业, 进一步结合企业数据库, 对 一阶控制价进行全面审查, 并根据审查意见出具二阶控制价; 在二阶控制价基础上, 通过组织专家评审会的形式将前面的 控制价形成进行详细说明和重点阐述, 通过专家评审修改后 形成三阶控制价; 将三阶控制价作为申报控制价向领导汇报 并组织进行控制价最终确认会签流程, 会签完成的控制价作 为最终项目招标控制价予以发布。通过上述四阶控制法, 最大 程度地逼近项目真实成本。

第二, 建立对基础设计单位及外部审查单位的绩效考核 和激励机制。为确保基础设计概算审查的合理性和准确性, 首 先,建立基础设计概算的第三方审查制度,即委托不低于基础 设计编制单位资质的造价咨询机构对基础设计的概算进行第 三方的审查; 其次, 建立基础设计编制单位与外部审查单位的 绩效考核机制, 即在基础设计编制单位服务费中预留考核费 用和奖励费用。项目后期施工图预算与基础设计概算造价相 差幅值在 $10 \%$ 以内, 基础设计编制单位获得全部考核费用, 在 5\%以内获得奖励费用。同时, 对基础设计审查单位设置同 样的绩效考核和激厉玑机制。

第三, 设置弹性的合同价款调节机制。对于基础设计概 算对应的目标成本小于签约合同价款目标成本时,建立分阶 调整机制。对于基础设计与签约合同价款成本差值在 5\%以 内时, 根据基础设计概算调整合同价款, 并保持合同价款中 的成本目标构成比例与批复基础设计保持一致; 差值在 5\% $10 \%$ 的部分, 在调整合同价款与批复基础设计一致的前提 下, 将固定酬金部分予以调高以补偿承包商部分合同额的损 失; 超过 $10 \%$ 的部分, 合同价款不予调整, 并将超过部分的差 值直接触发业主项目预备金，以预备金补偿投资控制差额。 对于基础设计概算对应的目标成本大于签约合同价款的目 标成本时,在保持签约合同总价不变的前提下,按照批复基 础设计调整签约合同价款中的目标成本构成, 同时, 建立酬 金计算的议价机制, 由承包商在固定比例酬金与结余分成上 报分配方案。

第四，建立重大设计变更的费用认定标准及成本分担机 制。在项目实施阶段, 建立对重大设计方案变更的审核及审批 制度, 从严控制重大设计变更, 同时, 对重大设计变更设置量 
化的认定标准,比如, 造成合同价款变化超过 $1 \%$ 或者合同价 款增加 100 万就可以认定为重大设计变更。同时,要建立重大 设计变更合同价款调整的评估与确认机制，对变更价款进行 重点审核。另外,对于被认定为重大设计变更导致的设备材料 采购费用及建筑安装费用, 工期延误造成的其他成本, 也应该 建立相应的责任认定和成本分担机制，确保双方高质量的继 续履行合同。

第五, 建立费用控制的联席会议制度, 确保质量、安全的 费用投人。首先, 在招标控制价的编制及基础设计概算审核阶 段, 要对质量及安全管理投入进行充分的考虑,邀请质量管理 部门及安全管理部门参与到费用的评审过程中。其次, 在合同 执行阶段, 要建立物资设备部门与质量部门的沟通机制, 确保 物资设备部门的要求与质量管理部门的监管要求一致, 尤其 是对设备材料型号及参数的要求要确保一致, 不能单方面提 高标准。最后,建立质量管理的奖罚制度与安全管理的专款专 用制度, 对每一期进度款设置质量考核金, 实行滚动管理, 在 下一期进度款中对上一期的预留考核金按照考核结果按时发 放。同时, 足额提取安全管理费用,建立安全管理投人的专款
专用账户,并进行监管, 确保安全管理费用的足额使用。

第六, 建立阶段性成本超支预警及结算罚金熔断机制。建 立阶段性的成本核算报告制度，承包商应业主要求及时将各 季度及截至目前发生的成本进行统计，并编制合同目标成本 完成分析报告, 系统分析目标成本的动态变化过程, 对报告出 现的超过阶段目标成本的行为要进行及时预警，协助承包商 进行成本控制, 严格控制超过目标成本行为的出现。对于确有 最终结算成本超过目标成本时, 也要建立超支熔断机制, 即对 于承包商结算合同成本超过目标成本的比例超过红线比例时 （如 30\%）, 超过部分处罚条款自动熔断失效(30\%以内的成本 按照合同约定进行罚款, 超过 $30 \%$ 的部分罚款制度失效), 业 主将择机启动预备费，用于补偿因为超支造成的投资增加。

\section{参考文献}

[1]刘鹏.建设项目承包合同计价方式分析[J].重庆建筑大学学报, 2004(6):103-105.

[2]陈权,吕爽.以成本加酬金作为计价方式的 EPC 总承包合同研 究[J].项目管理技术,2014(12):70-74.

[3]王延树,成虎.EPC 总承包新型计价合同—目标合同应用研 究[J].重庆建筑大学学报,2008(5):73-76. 